University of Nebraska - Lincoln

DigitalCommons@University of Nebraska - Lincoln

Robert Streubel Papers

Research Papers in Physics and Astronomy

4-22-2019

\title{
Origin of enhanced anisotropy in FePt-C granular films revealed by XMCD
}

Robert Streubel

Lawrence Berkeley National Laboratory, streubel@unl.edu

Alpha T. N'Diaye

Lawrence Berkeley National Laboratory

Kumar Srinivasan

Western Digital

Antony Ajan

Western Digital

Peter Fischer

Lawrence Berkeley National Laboratory

Follow this and additional works at: https://digitalcommons.unl.edu/physicsstreubel

Part of the Atomic, Molecular and Optical Physics Commons, Condensed Matter Physics Commons, and the Other Physics Commons

Streubel, Robert; N'Diaye, Alpha T.; Srinivasan, Kumar; Ajan, Antony; and Fischer, Peter, "Origin of enhanced anisotropy in FePt-C granular films revealed by XMCD" (2019). Robert Streubel Papers. 8.

https://digitalcommons.unl.edu/physicsstreubel/8

This Article is brought to you for free and open access by the Research Papers in Physics and Astronomy at DigitalCommons@University of Nebraska - Lincoln. It has been accepted for inclusion in Robert Streubel Papers by an authorized administrator of DigitalCommons@University of Nebraska - Lincoln. 


\section{Origin of enhanced anisotropy in FePt-C granular films revealed by XMCD}

Cite as: Appl. Phys. Lett. 114, 162401 (2019); https://doi.org/10.1063/1.5092719

Submitted: 13 February 2019 • Accepted: 05 April 2019 • Published Online: 23 April 2019

Robert Streubel, (D) Alpha T. N'Diaye, Kumar Srinivasan, et al.

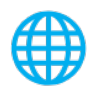

\section{ARTICLES YOU MAY BE INTERESTED IN}

Review Article: FePt heat assisted magnetic recording media

Journal of Vacuum Science \& Technology B 34, 060801 (2016); https://

doi.org/10.1116/1.4965980

Néel vector reorientation in ferromagnetic/antiferromagnetic complex oxide nanostructures Applied Physics Letters 114, 192403 (2019); https://doi.org/10.1063/1.5094604

Growth of $\mathrm{L1}_{0}$-ordered crystal in FePt and FePd thin films on $\mathrm{MgO}(001)$ substrate AIP Advances 6, 085302 (2016); https://doi.org/10.1063/1.4960554

\section{Challenge us.}

What are your needs for periodic signal detection?

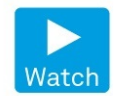

- Zurich

- Instruments

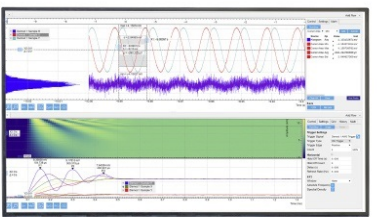

(- 


\title{
Origin of enhanced anisotropy in FePt-C granular films revealed by XMCD
}

\author{
Cite as: Appl. Phys. Lett. 114, 162401 (2019); doi: 10.1063/1.5092719 \\ Submitted: 13 February 2019 . Accepted: 5 April 2019 . \\ Published Online: 23 April 2019

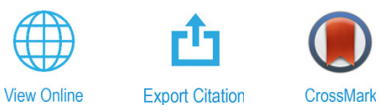

Robert Streubel, ${ }^{1, \text { a) }}$ (D) Alpha T. N'Diaye, ${ }^{2}$ (D) Kumar Srinivasan, ${ }^{3}$ Antony Ajan, ${ }^{3}$ and Peter Fischer ${ }^{1,4}$

\author{
AFFILIATIONS \\ ${ }^{7}$ Materials Sciences Division, Lawrence Berkeley National Laboratory, Berkeley, California 94720, USA \\ ${ }^{2}$ Advanced Light Source, Lawrence Berkeley National Laboratory, Berkeley, California 94720, USA \\ ${ }^{3}$ Western Digital, San Jose, California 95131, USA \\ ${ }^{4}$ Physics Department, UC Santa Cruz, Santa Cruz, California 95064, USA

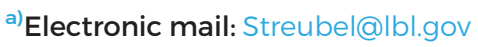

\begin{abstract}
We study the effect of carbon segregants on the spin and orbital moments of $L 1_{0}$ FePt granular media using x-ray magnetic circular dichroism (XMCD) spectroscopy and report an effective decoupling of the structural film properties from the magnetic parameters of the grains. The carbon concentration reduces the grain size from $(200 \pm 160) \mathrm{nm}^{2}$ down to $(50 \pm 20) \mathrm{nm}^{2}$ for 40 mol. \%C and improves sphericity and the order of grains, while preserving the crystalline order, spin and orbital moments, and perpendicular magnetocrystalline anisotropy. We identify the primary cause of enhanced saturation and coercive fields as the reduced demagnetization fields of individual grains. The ability to shrink grains without impairing their magnetic properties is a critical requirement for the commercialization of Heat-Assisted Magnetic Recording.
\end{abstract}

Published under license by AIP Publishing. https://doi.org/10.1063/1.5092719

Hard-disk drives utilizing magnetic recording technology are the dominant auxiliary storage devices owing to their capacity and price advantages. Within the next few years, magnetic recording technology is expected to transition from perpendicular magnetic recording (PMR) to energy assisted technologies, such as heat-assisted magnetic recording (HAMR). ${ }^{1}$ For these applications, the $L 1_{0}$ phase of $\mathrm{FePt}$ offering a very high magnetocrystalline anisotropy of $7 \mathrm{MJ} / \mathrm{m}^{3}$ is the material system of choice. ${ }^{2,3}$ The high anisotropy allows for pushing the smallest thermally stable grain sizes down to (3-4) nm, which corresponds to a storage density of several Tbits/in ${ }^{2}$. The writing process has to be energy assisted to overcome the high anisotropy at room temperature, e.g., facilitating laser induced heating of the FePt media above its Curie temperature. Magnetically isolated granular FePt films are typically sputter deposited alongside other constituents that preferentially form grain boundary phases or segregants. ${ }^{3-7}$ These constituents include carbon, oxides, nitrides, and carbides. Aside from segregants, dopants such as $\mathrm{Ag}$ and $\mathrm{Cu}$ may be introduced into the FePt to control the structural ordering temperature, Curie temperature, and other properties. A partial FePt/segregant phase separation can result in intermixing at the interface between the two. For instance, a core-shell type of microstructure with an $\mathrm{FeO}_{x}$ shell surrounding an FePt grain has been observed in $\mathrm{FePt}-\mathrm{SiO}_{2}$ systems. ${ }^{8}$ While it is well understood that segregants and dopants in the FePt system strongly influence the magnetic bit recording parameters, ${ }^{3-7}$ little is known about their effect on spin and orbital moments, and demagnetization field contributions.

In this work, we investigate the effect of carbon segregants on the spin and orbital Fe moments of $L 1_{0}$ FePt nanogranular media and determine the relation of enhanced coercive and saturation fields to the grain size. The spin and orbital moments are quantified by x-ray absorption spectroscopy (XAS) and x-ray magnetic circular dichroism (XMCD) spectroscopy. The carbon promotes sphericity and a narrow size distribution of the magnetic grains, which increase saturation and coercive fields due to reduced demagnetization fields, while preserving spin and orbital moments and perpendicular magnetocrystalline anisotropy. The FePt films were prepared at Western Digital following the procedure described in Ref. 8 . We note in particular the following: (1) The FePt-C films were deposited by cosputtering from separate FePt and $\mathrm{C}$ targets. Three sets of $5 \mathrm{~nm}$-thick films were grown in situ at $400^{\circ} \mathrm{C}, 500^{\circ} \mathrm{C}$, and $600^{\circ} \mathrm{C}$ substrate temperatures. We additionally grew $10 \mathrm{~nm}$-thick sister samples to minimize the experimental uncertainty of crystallographic analysis. (2) We use a $4 \mathrm{~nm}$-thick Co capping layer deposited at room temperature to facilitate the complete reversal of the moments during the XAS/XMCD experiments. Without the Co capping layer, the switching fields for the ordered FePt films in this study are well beyond $30 \mathrm{kOe}$ and not accessible in this experiment. 

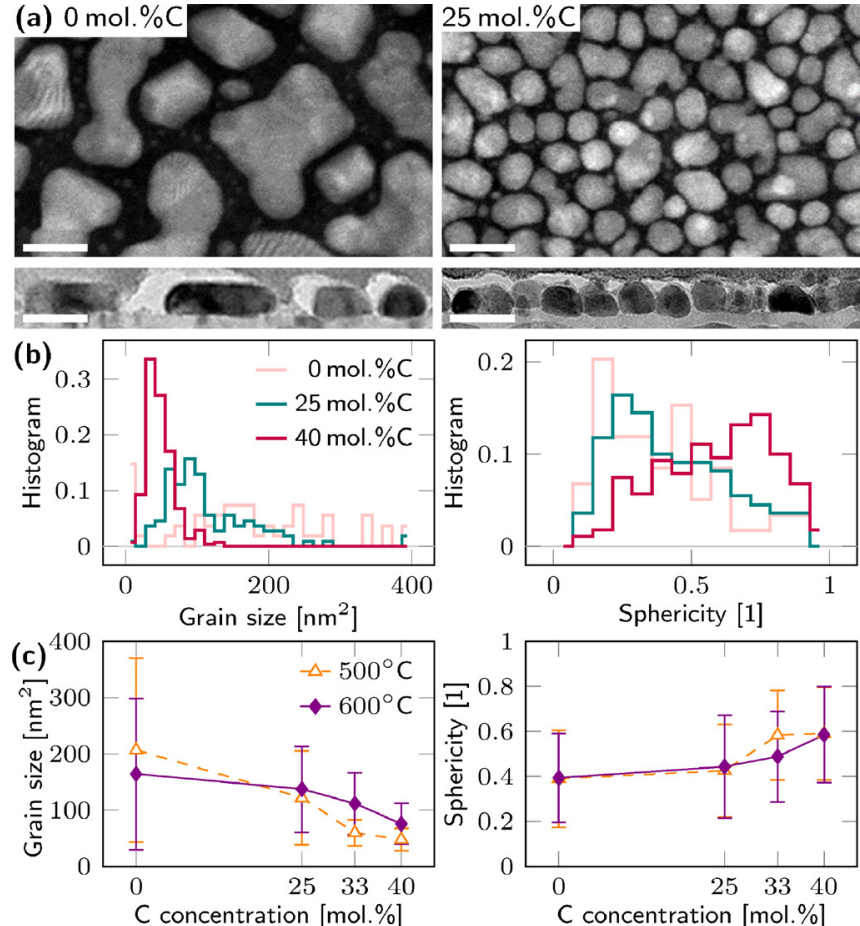

FIG. 1. Structural modification of $L 1_{0}$ FePt granular microstructures by addition of carbon segregants. (a) Top and cross-sectional view of the $10 \mathrm{~nm}$-thick FePt film grown at $600^{\circ} \mathrm{C}$ recorded in the STEM-mode and bright-field mode of TEM, respectively. Scale bars are $20 \mathrm{~nm}$. (b) Distribution of the grain size and sphericity quantifying transformation from large, irregular to small, circular grains. (c) $\mathrm{C}$ concentration dependence of the mean grain size and shape for different growth temperatures.

The Co layer solely serves as a switching-assist layer, so that the samples can be saturated in the available fields, and affects neither the morphology nor magnetic properties of the FePt-C system.

The structural properties of the granular films without the Co capping layer are probed using scanning electron microscopy with an in-lens and an electron backscatter detector as well as scanning transmission electron microscopy and bright-field transmission electron microscopy of top and cross-sectional views, respectively. Each sample represents a discontinuous, granular film as exemplarily shown for specimens grown at $600^{\circ} \mathrm{C}$ [Fig. 1(a)]. The grain morphology is quantified by the distribution of the grain size and shape (sphericity) [Fig. 1(b)], which reveals a systematic linear decrease (increase) in the grain size (sphericity) with the increasing carbon concentration. In particular, the grain size of FePt is reduced from $(200 \pm 160) \mathrm{nm}^{2}$ down to $(50 \pm 20) \mathrm{nm}^{2}$ for the $40 \mathrm{~mol}$. \%C concentration [Fig. 1(c)]. Note that these values may vary with the FePt film thickness. The experimental uncertainty is given by the standard deviation of the corresponding distributions. The sphericity is declared as the ratio of the two major axes of individual grains approximated as ellipses. While specimens grown at $500^{\circ} \mathrm{C}$ and $600{ }^{\circ} \mathrm{C}$ behave qualitatively similarly in view of the film morphology [Fig. 1(c)], a growth temperature of $40{ }^{\circ} \mathrm{C}$ leads to a maze microstructure which prevails for carbon concentrations up to $40 \mathrm{~mol}$. \%.

The carbon driven morphology change preserves the crystalline $L 1_{0}$ structure for concentrations up to 40 mol. \% (30 vol.\%) [Fig. 2(a)]
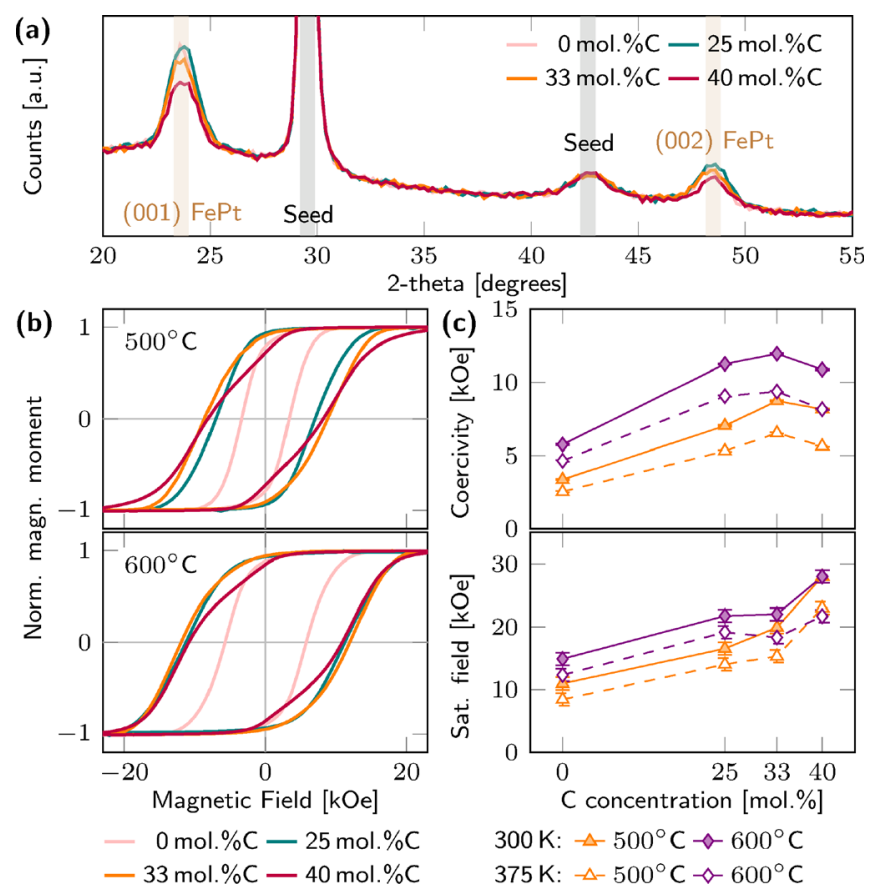

FIG. 2. Preservation of crystallinity and enhancement of coercive and saturation fields along the easy axis with increasing carbon concentration. (a) Two-theta x-ray diffraction scans of the $10 \mathrm{~nm}$-thick FePt film confirming crystallographic properties of $L 1_{0}$ FePt. (b) Room temperature out-of-plane magnetic hysteresis loops recorded by a Physical Property Measurement System. (c) Coercive and saturation fields of samples deposited at $500^{\circ} \mathrm{C}$ and $600^{\circ} \mathrm{C}$, measured at $300 \mathrm{~K}$ and $375 \mathrm{~K}$. Magnetic data in (b) and (c) are taken from Co capped $5 \mathrm{~nm}$-thick films.

as evident from a nearly constant rocking curve width of $\left(6.2^{\circ} \pm 0.2^{\circ}\right)$, and improves the overall surface roughness from $1.2 \mathrm{~nm}(0 \mathrm{~mol} . \% \mathrm{C})$ to $0.6 \mathrm{~nm}(>25 \mathrm{~mol} . \% \mathrm{C})$. However, it strongly affects the saturation field and coercivity, which increases for concentrations from 0 to $33 \mathrm{~mol}$. \%C (Fig. 2). The decrease in coercivity at $40 \mathrm{~mol}$. \%C coincides with the lowered (002) diffraction peak intensity. Despite similarities, we like to emphasize that the magnetics and x-ray diffraction data are taken from Co capped $5 \mathrm{~nm}$-thick FePt films and $10 \mathrm{~nm}$-thick sister samples without capping, respectively. Interestingly, the relative change of both fields as a function of carbon concentration is similar for different measurement temperatures and particularly growth temperatures despite distinct values for FePt with 0 mol. \%C. This behavior suggests a governing role of extrinsic properties, i.e., grain morphology, not crystallinity, as discussed in detail below. The dependence on growth temperature originates from a slightly better crystalline ordering for specimens deposited at $600^{\circ} \mathrm{C}$. The percentage of switched grains in specimens with $40 \mathrm{~mol}$. \%C at remanence is similar to that of pure FePt of roughly $10 \%$. Note that the lack of a fully saturated remanent state and relatively low coercive/saturation fields (thrice less with Co capping) are due to the exchange-spring reversal caused by coupling to the Co capping layer. A similar carbon concentration dependence of the coercive field peaking at $30 \mathrm{~mol}$. \% was observed in previous works and assigned to an improved crystallinity. ${ }^{3,4}$

Further insight into chemical and magnetic properties is given by XAS and XMCD spectroscopy performed at beamline 6.3.1 at the 

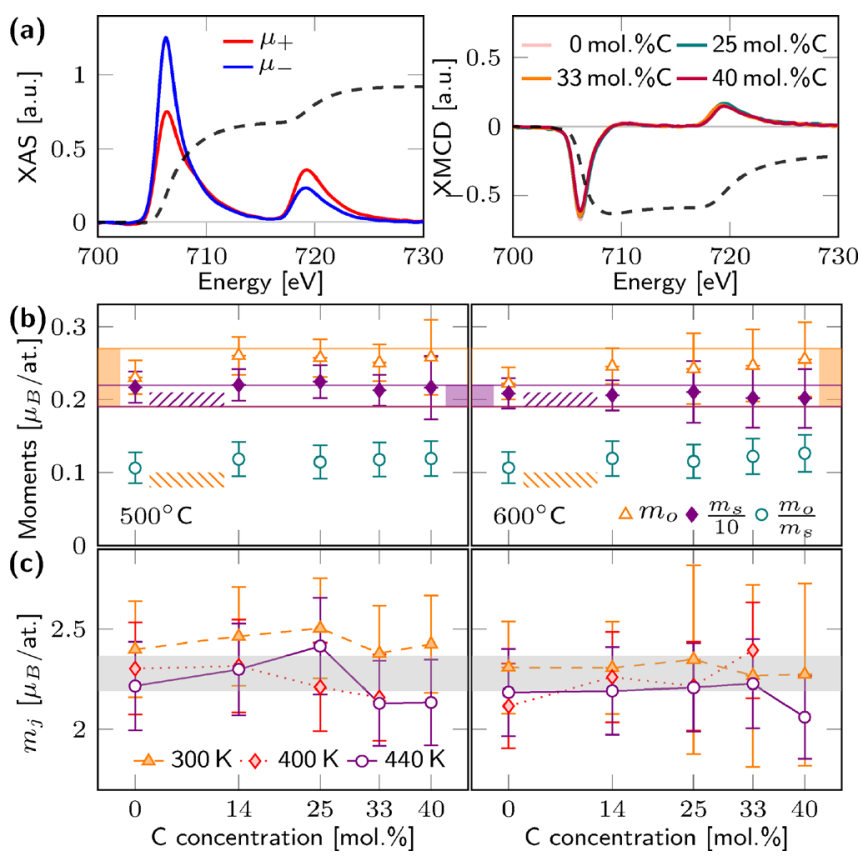

FIG. 3. Quantification of spin and orbital moments utilizing x-ray magnetic circular dichroism spectroscopy. (a) X-ray absorption spectra and XMCD signal near Fe $L_{3,2}$ edges shown for $L 1_{0}$ FePt grown at $500^{\circ} \mathrm{C}$ and saturated out-of-plane in $\pm 19 \mathrm{kOe}$. Dashed lines plot the integrals used for quantitative analysis as defined in the text. (b) Room temperature spin and orbital moments as well as their ratio as a function of carbon concentration. Colored horizontal lines indicate literature values for Fe moments in $L 1_{0}$ FePt. Striped lines refer to bcc Fe moments. (c) Total moments plotted for various measurement temperatures and carbon concentrations.

Advanced Light Source and quantifying spin and orbital moments to assess the governing magnetocrystalline anisotropy. The XAS near the Fe $L_{3,2}$ edges [ $\left.(690-750) \mathrm{eV}\right]$ are recorded in the presence of an external magnetic field ( $\pm 19 \mathrm{kOe})$ normal to the sample surface. The spectra plotted in Fig. 3(a) are typical for metallic FePt and confirm that carbon solely affects the film morphology but not the chemistry as it migrates to the grain boundaries instead of intercalation. For comparison, FePt specimens with $50 \mathrm{~mol}$. \%C reveal an obvious shoulder of the $L_{3}$ absorption peak associated with $\mathrm{Fe}-\mathrm{C}$ hybridization and a carbon intercalation. Despite the high sensitivity of XAS to chemical bonding, it is impossible to completely exclude hybridization at $40 \mathrm{~mol}$. \%C. However, it does not measurably impact the magnetic moments. This interpretation is consistent with micromagnetic simulations of the effect of intercalated carbon inside $L 1_{0}$ FePt that leads to a fading of magnetocrystalline anisotropy at $10 \mathrm{~mol} . \% \mathrm{C}$ and beyond. ${ }^{3}$ Calculating the integrals of both XAS and XMCD spectra $r$ $=\int_{L_{3}+L_{2}}\left(\mu_{+}+\mu_{-}\right) \mathrm{d} \omega, q=\int_{L_{3}+L_{2}}\left(\mu_{+}-\mu_{-}\right) \mathrm{d} \omega, p=\int_{L_{3}}\left(\mu_{+}-\mu_{-}\right) \mathrm{d} \omega$, shown as dashed curves in Fig. 3(a), allows for quantifying the orbital and spin moment using the sum rules ${ }^{9,10}$ according to $m_{o}=-\frac{4 q}{3 r} n_{h}$ and $m_{s}=-\frac{6 p-4 q}{r} n_{h}\left(1+\frac{7\left\langle T_{z}\right\rangle}{2\left\langle S_{z}\right\rangle}\right)^{-1}$, respectively. The Fe electron hole density is set to $n_{h}=3.73$ according to first-principles calculations. ${ }^{11}$ Note that the expectation value of the magnetic dipole operator $\left\langle T_{z}\right\rangle$ is constant for all investigated systems because of identical film thicknesses. Additionally, the $L 1_{0}$ crystal structure of FePt causes a substantial normal spin-orbit coupling that nullifies surface contributions. Hence, contributions from the magnetic dipole operator are neglected to simplify the study of the impact of the carbon concentration. The accordingly obtained data reveal significantly larger orbital moments than bcc Fe $\mathrm{e}^{12}$ due to spin-orbit coupling with adjacent $\mathrm{Pt}$ layers and similar values for spin moments [Fig. 3(b)]. In fact, the experimental data agree well with the range of moments reported in previous works on $L 1_{0} \mathrm{FePt}$ and $L 1_{0}$ FePt-C..$^{3,7,13,14}$ Overall, the spin moment is within experimental uncertainty unaffected by the carbon concentration affirming the absence of chemical disorder or a deadlayer surrounding each grain. The slightly increased orbital moment, virtually the same for any investigated carbon concentration, indicates an enhanced vertical ordering of the $\mathrm{Pt} / \mathrm{Fe}$ layer system compared with pure $L 1_{0} \mathrm{FePt}$, which does not further improve with the carbon concentration. For specimens grown at $600^{\circ} \mathrm{C}$, the saturation field even with the Co capping layer exceeds at room temperature the experimentally accessible value of $19 \mathrm{kOe}$ leading to a $90 \%$ saturated sample (Fig. 2). This partial saturation is taken into account by scaling both orbital and spin moments, which induces a slightly larger uncertainty compared with other measurements. Furthermore, the strong spin-orbit coupling and the corresponding perpendicular magnetocrystalline anisotropy of the $L 1_{0}$ FePt films suppress an obvious temperature dependence in the probed range (300-440) K [Fig. 3(c)]. Note that both statements on the carbon concentration and temperature apply analogously to the magnetocrystalline anisotropy due to its direct relation to the ordering of orbital moments.

Consequently, the enhancement of coercive and saturation fields has to originate from the change in the grain morphology. To confirm this assertion, we compare the contributions of demagnetization and magnetocrystalline anisotropy energy to the overall energy. The anisotropy energy $E$ associated with the switching of a magnetic grain can be calculated via $E=\mu_{0} \int_{e . a} M \mathrm{~d} H-\mu_{0} \int_{h \cdot a} M \mathrm{~d} H$ of hysteresis-free curves along the easy and hard axes. Note that the significant magnetocrystalline anisotropy of the $L 1_{0}$ FePt film does not allow for saturating the film along the hard axis. Hence, we approximated the relative change of the energy $E$ as a function of grain size (carbon concentration) from the easy axis hysteresis loops as $E \approx \frac{1}{2} \mu_{0} M_{s} H_{s}$ assuming a similar relative change for saturation fields $H_{s}$ along hard and easy axes. Furthermore, the magnetic energy $E \approx E_{d}+E_{u}$ comprises demagnetization energy $E_{d}=\frac{1}{2} \mu_{0} M_{s}^{2} V \mathbf{m}^{\mathrm{T}} \mathbf{N m} \approx \alpha N_{a}$ and the magnetocrystalline anisotropy energy $E_{u}=\beta K_{u}$, favoring easy plane and easy axis anisotropy, respectively. Here, $\mathbf{m}$ is the unit magnetization vector taking the value $(0,0,1)$ for perpendicularly magnetized grains and $\mathbf{N}$ is the demagnetization tensor. The corresponding demagnetization factor $N_{a}$ perpendicular to the substrate surface is defined by $N_{a}=\frac{1}{2} a b c \int_{0}^{\infty} \frac{\mathrm{d} x}{\left(a^{2}+x\right) \sqrt{\left(a^{2}+x\right)\left(b^{2}+x\right)\left(c^{2}+x\right)}}$ with major axes $a, b$, and $c .{ }^{15}$ In this notation, $a$ is equal to half the film thickness $(2.5 \mathrm{~nm})$. The grains are approximated as rotation ellipsoids with a circular lateral expansion ( $b=c=\sqrt{A / \pi})$, which slightly underestimates $N_{a}$ for large grains. The grain size $A$ is taken from Fig. 1(c). The coefficients $\alpha$ and $\beta$ denote quantities, i.e., volume $V$ and saturation magnetization $M_{s}$, which are in first approximation constant throughout the sample series and therefore irrelevant to the following estimation. As evident from Fig. 4 (a), the carbon concentration of the $5 \mathrm{~nm}$-thick films significantly alters the demagnetization field owing to a shrinking of the grains 

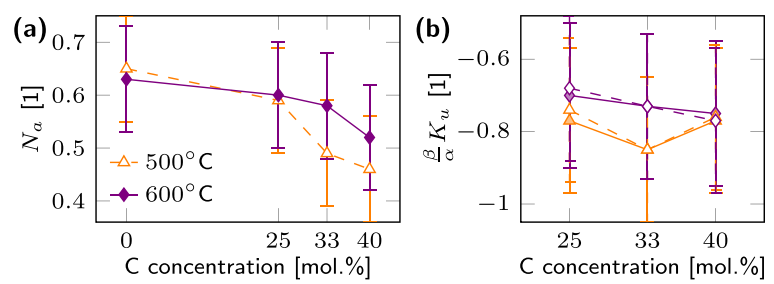

$300 \mathrm{~K}:-500^{\circ} \mathrm{C} \diamond-600^{\circ} \mathrm{C}$ $375 \mathrm{~K}:-\triangle-500^{\circ} \mathrm{C} \diamond-600^{\circ} \mathrm{C}$

FIG. 4. Indirect confirmation of the dominant role of shape anisotropy. (a) Normal component of demagnetization tensor estimated for rotation ellipsoid approximation. (b) Normalized magnetocrystalline anisotropy with nearly constant values indicating the governing role of shape anisotropy.

down to nearly isotropic expansion (1/3). The increasingly relevant demagnetization factor combined with the grain size distribution explains why the slope of the hysteresis becomes more shallow at large carbon concentrations [Fig. 2(a)]. The demagnetization field contribution governs the relative change of the saturation fields with respect to pure FePt,

$$
\frac{H_{s}^{j}}{H_{s}^{0}} \approx \frac{\alpha N_{a}^{j}+\beta K_{u}}{\alpha N_{a}^{0}+\beta K_{u}},
$$

which is confirmed by calculating $\frac{\beta}{\alpha} K_{u}$ [Fig. 4(b)]. Note that the sole purpose of this estimate is to verify or refute a carbon dependence of the normalized anisotropy $\frac{\beta}{\alpha} K_{u}$ after subtracting contributions of grain size dependent demagnetization fields. It does not serve as a quantification of the anisotropy itself. The negative sign is caused by counteracting magnetocrystalline and demagnetization field favoring easy axis and easy plane anisotropy, respectively. Within experimental uncertainty, primarily originating from the relatively broad grain size distribution [Fig. 1(c)] and underestimated demagnetization fields for large grains, all samples reveal the same $\frac{\beta}{\alpha} K_{u}$ value indicating a modification of the magnetic properties primarily driven by the grain morphology. This result implies that the current understanding of the role of carbon, enhancing coercivity and anisotropy due to improved crystallinity and hence magnetocrystalline anisotropy, is by itself insufficient.

In conclusion, we showed that the carbon concentration in $L 1_{0}$ FePt granular microstructures significantly improves structural properties: it promotes sphericity and a narrow size distribution of the magnetic grains. This microstructure supports enhanced saturation and coercive fields due to reduced demagnetization fields, while preserving, up to $40 \mathrm{~mol}$. \% , spin and orbital moments and perpendicular magnetocrystalline anisotropy. Higher carbon concentrations cause atomic intermixing impairing structural and magnetic quality. While specimens grown at $500^{\circ} \mathrm{C}$ show a more prominent structural change compared with $600^{\circ} \mathrm{C}$, the latter exhibit larger saturation and coercive field values due to the superior crystalline order. In contrast, $400^{\circ} \mathrm{C}$ growth temperature leads to a maze microstructure. We found that carbon segregates at the grain boundaries, thereby reducing in the present case of $5 \mathrm{~nm}$-tick $L 1_{0}$ FePt the grain size from $(200 \pm 160) \mathrm{nm}^{2}$ down to $(50 \pm 20) \mathrm{nm}^{2}$. The accordingly altered demagnetization field of individual grains is the primary cause of an increased effective perpendicular magnetic anisotropy in contrast to the current understanding of an improved crystallinity and magnetocrystalline anisotropy.

This work was funded by the U.S. Department of Energy, Office of Science, Basic Energy Sciences, Materials Sciences and Engineering Division, under Contract No. DE-AC02-05-CH11231 within the NEMM program (MSMAG). This research used resources of the Advanced Light Source, which is a DOE Office of Science User Facility under Contract No. DE-AC02-05CH11231.

\section{REFERENCES}

${ }^{1}$ D. Weller, G. Parker, O. Mosendz, E. Champion, B. Stipe, X. Wang, T. Klemmer, G. Ju, and A. Ajan, IEEE Trans. Magn. 50, 1 (2014).

${ }^{2}$ D. Weller, O. Mosendz, G. Parker, S. Pisana, and T. S. Santos, Phys. Status Solidi A 210, 1245 (2013).

${ }^{3}$ J. Wang, H. Sepehri-Amin, H. Tajiri, T. Nakamura, K. Masuda, Y. Takahashi, T. Ina, T. Uruga, I. Suzuki, Y. Miura, and K. Hono, Acta Mater. 166, 413 (2019).

${ }^{4}$ A. Perumal, Y. K. Takahashi, and K. Hono, J. Appl. Phys. 105, 07 B732 (2009).

5. Chen, J. Hu, B. Lim, Y. Ding, G. Chow, and G. Ju, IEEE Trans. Magn. 45, 839 (2009).

${ }^{6}$ S. Pisana, O. Mosendz, G. J. Parker, J. W. Reiner, T. S. Santos, A. T. McCallum, H. J. Richter, and D. Weller, J. Appl. Phys. 113, 043910 (2013).

${ }^{7}$ S. Sakamoto, K. Srinivasan, R. Zhang, O. Krupin, K. Ikeda, G. Shibata, Y. Nonaka, Z. Chi, M. Sakamaki, K. Amemiya, A. Fujimori, and A. Ajan, Phys. Rev. B 96, 144437 (2017).

${ }^{8}$ Y. Zhang, A. Kalitsov, J. Ciston, O. Mryasov, B. Ozdol, J. Zhu, S. Jain, B. Zhang, B. Livshitz, A. Chernyshov, A. Ajan, P. Dorsey, G. Bertero, R. Acharya, A. Greene, and S. Myers, AIP Adv. 8, 125018 (2018).

${ }^{9}$ B. T. Thole, P. Carra, F. Sette, and G. van der Laan, Phys. Rev. Lett. 68, 1943 (1992).

${ }^{10}$ P. Carra, B. T. Thole, M. Altarelli, and X. Wang, Phys. Rev. Lett. 70, 694 (1993).

${ }^{11}$ S. Miwa, M. Suzuki, M. Tsujikawa, K. Matsuda, T. Nozaki, K. Tanaka, T. Tsukahara, K. Nawaoka, M. Goto, Y. Kotani, T. Ohkubo, F. Bonell, E. Tamura, K. Hono, T. Nakamura, M. Shirai, S. Yuasa, and Y. Suzuki, Nat. Commun. 8, 15848 (2017).

${ }^{12}$ C. T. Chen, Y. U. Idzerda, H.-J. Lin, N. V. Smith, G. Meigs, E. Chaban, G. H. Ho, E. Pellegrin, and F. Sette, Phys. Rev. Lett. 75, 152 (1995).

${ }^{13}$ O. Dmitrieva, M. Spasova, C. Antoniak, M. Acet, G. Dumpich, J. Kästner, M. Farle, K. Fauth, U. Wiedwald, H.-G. Boyen, and P. Ziemann, Phys. Rev. B 76, 064414 (2007).

${ }^{14}$ D. Xu, C. Sun, J. Chen, S. Heald, B. Sanyal, R. Rosenberg, T. Zhou, and G. Chow, J. Phys. D: Appl. Phys. 48, 255001 (2015).

${ }^{15}$ A. Hubert and R. Schäfer, Magnetic Domains (Springer, 1998). 